\title{
A new multiplex PCR-based reverse line-blot hybridization (mPCR/RLB) assay for rapid staphylococcal cassette chromosome mec (SCCmec) typing
}

Correspondence Gwendolyn L. Gilbert l.gilbert@usyd.edu.au

Received 12 November 2008 Accepted 5 May 2009

\section{Lin Cai, ${ }^{1}$ † Fanrong Kong, ${ }^{2}$ † Oinning Wang, ${ }^{2}$ Huiping Wang, ${ }^{3}$ Meng Xiao, ${ }^{4}$ Vitali Sintchenko ${ }^{2,5}$ and Gwendolyn L. Gilbert ${ }^{2,5}$}

\author{
${ }^{1}$ Department of Dermatology, Peking University People's Hospital, Beijing 100044, PR China \\ ${ }^{2}$ Centre for Infectious Diseases and Microbiology, Institute of Clinical Pathology and Medical \\ Research (ICPMR), Westmead, New South Wales, Australia \\ ${ }^{3}$ Department of Dermatology, Tianjin Medical University General Hospital, Tianjin, PR China \\ ${ }^{4}$ Life Science College, Peking University, Beijing, PR China \\ ${ }^{5}$ Western Clinical School, University of Sydney, Sydney, Australia
}

\begin{abstract}
The aim of this study was to develop a new discriminatory method for MRSA SCCmec typing based on multiplex PCR-based reverse line-blot hybridization (mPCR/RLB) assay to enable rapid identification and classification of MRSA SCCmec types in a clinical laboratory. Forty-five primer sets and 49 probes were designed and tested in uniplex PCR (UPCR) and mPCR/RLB. Probes were compared in silico to 14 whole-genome sequences and 18 partial SCCmec gene sequences of Staphylococcus aureus and complete genome and partial SCCmec genes of seven non-MRSA strains, including meticillin-susceptible $S$. aureus and meticillin-resistant coagulasenegative staphylococci. The method was tested on a set of 42 well-characterized reference MRSA strains. It identified all five epidemiologically relevant SCCmec types and 26 subtypes, including established and new subtypes of SCCmec III, IV (eight subtypes each) and V (three subtypes). The discriminatory power of mPCR/RLB SCCmec typing was similar to that of MLST and spa typing (Simpson indices of diversity of 0.916, 0.926 and 0.882, respectively; differences not statistically significant). The application of mPCR/RLB hybridization assay to MRSA SCCmec typing can improve the specificity, discriminatory power and throughput of the typing procedure. The detection of up to $43 \mathrm{mPCR}$ products in a single hybridization assay transforms MRSA $\mathrm{SCC} m e c$ typing from passive epidemiological library typing into a potential tool for near-real-time infection control surveillance and tracking of MRSA transmission in hospitals.
\end{abstract}

\section{INTRODUCTION}

Morbidity and mortality from meticillin-resistant Staphylococcus aureus (MRSA) infections and the emerging threat of community-associated MRSA highlight the importance of timely surveillance (Aires de Sousa \& de Lencastre, 2004; Ma et al., 2002). Meticillin resistance is conferred by the mecA gene, which is carried on a mobile genetic element known as the staphylococcal cassette chromosome mec (SCCmec) (Ito \& Hiramatsu, 1998; Ito

†These authors contributed equally to this work.

Abbreviations: mPCR/RLB, multiplex PCR-based reverse line-blot hybridization; MLST, multilocus sequence typing; MRSA, meticillinresistant S. aureus; MSSA, meticillin-sensitive S. aureus; SCCmec, staphylococcal cassette chromosome mec; uPCR, uniplex PCR. et al., 1999). The combination of mec complex class and cassette chromosome recombinase ( $c c r)$ genes determines the SCCmec type (Ito et al., 2004; Kondo et al., 2007). Currently, seven SCCmec types (I-VII) and a number of subtypes have been described, ranging in size from 20.9 to 66.9 kb (Ito et al., 2001, 2004; Daum et al., 2002; Oliveira et al., 2006a; Takano et al., 2008). SCCmec types I and IVVII are usually resistant only to $\beta$-lactam antibiotics, while types II and III are often resistant to multiple classes of antibiotics due to additional resistance genes integrated into SCCmec (Deurenberg \& Stobberingh, 2008). MRSA SCCmec types I-III have been linked with healthcareassociated infections (Ito et al., 2001) whereas communityacquired MRSA infections are typically caused by SCCmec types IV, V or VII (Ito et al., 2004; Daum et al., 2002; Takano et al., 2008). Evidence has been emerging of 
Table 1. Strains of 42 well-characterized meticillin-resistant $S$. aureus isolates used in the study

\begin{tabular}{|c|c|c|c|}
\hline ID & Strain & spa type* & Clonal type $\dagger$ \\
\hline 1 & FH53末 & $\mathrm{t} 127$ & ST 1-SCCmec-I \\
\hline 2 & COLS & t008 & ST250-SCCmec-I \\
\hline 3 & BK2464§ & t002 & ST5-SCCmec-II \\
\hline 4 & E822485末 & t018 & ST36-SCCmec-II \\
\hline 5 & RPAH $18 \ddagger$ & t037 & ST239-SCCmec-III \\
\hline 6 & RPAH15末 & t037 & ST239-SCCmec-III \\
\hline 7 & HDG2\$ & $\mathrm{t} 421$ & ST239-SCCmec-IIIB \\
\hline 8 & $14176-5710 \ddagger$ & t1959 & ST 239-SCCmec-III \\
\hline 9 & RDH81末 & t037 & ST239-SCCmec-IIIA \\
\hline 10 & AH1末 & t037 & ST128-SCCmec-IIIA \\
\hline 11 & AH13末 & $\mathrm{t} 037$ & ST239-SCCmec-IIIA \\
\hline 12 & HU25§ & $\mathrm{t} 138$ & ST239-SCCmec-IIIA \\
\hline 13 & K704540末 & $\mathrm{t} 037$ & $\mathrm{ST} \sim 239-\mathrm{SCC} m e c-\mathrm{III}$ \\
\hline 14 & K711532‡ & t037 & ST $\sim 239$-SCCmec-III \\
\hline 15 & ANS46\$ & $\mathrm{t} 037$ & ST239-SCCmec-III \\
\hline 16 & PC $8 \ddagger$ & $\mathrm{t} 127$ & ST1-SCCmec-IV \\
\hline 17 & FH43‡ & $\mathrm{t} 127$ & $\mathrm{ST} \sim 1$-SCCmec-IV \\
\hline 18 & RPH 85 & $\mathrm{t} 127$ & ST $\sim 1-S C C m e c-I V$ \\
\hline 19 & RHH10 & $\mathrm{t} 127$ & $\mathrm{ST} \sim 1$-SCCmec-IV \\
\hline 20 & B8-10末 & t711 & $\mathrm{ST} \sim 8$-SCCmec-IV \\
\hline 21 & PAH $58 \ddagger$ & t019 & ST30-SCCmec-IV \\
\hline 22 & E804531末 & t002 & ST5-SCCmec-IV \\
\hline 23 & CH97‡ & T002 & ST73-SCCmec-IV \\
\hline 24 & RBH98 & $\mathrm{t} 202$ & ST93-SCCmec-IV \\
\hline 25 & $13792-4492 \ddagger$ & $\mathrm{t} 202$ & ST $93-\mathrm{SCC} m e c-\mathrm{IV}$ \\
\hline 26 & IP01M1081‡ & $\mathrm{t} 216$ & ST59-SCCmec-IV \\
\hline 27 & MW2S & $\mathrm{t} 128$ & ST1-SCCmec-IV \\
\hline 28 & CH16末 & $\mathrm{t} 032$ & ST22-SCCmec-IV \\
\hline 29 & $\mathrm{CH} 69 \ddagger$ & $\mathrm{t} 1963$ & ST 22-SCCmec-IV \\
\hline 30 & DEN2988\$ & t008 & ST8-SCCmec-IVA \\
\hline 31 & F829549‡ & $\mathrm{t} 186$ & ST88-SCCmec-IV \\
\hline
\end{tabular}

potential associations between MRSA genotype and clinical outcomes (Lalani et al., 2008).

SCCmec typing and subtyping provides an opportunity to detect and reduce the transmission of MRSA in healthcare settings (Ito et al., 2004; Kondo et al., 2007). Several multiplex PCR (mPCR)-based methods have been described to determine the structure of the SCCmec complex (Oliveira \& de Lencastre, 2002; Zhang et al., 2005; Kondo et al., 2007; Milheirico et al., 2007) but most require several reactions, produce long amplicons and are time consuming (Chongtrakool et al., 2006; Kondo et al., 2007), making them impractical for outbreak investigations or early-warning systems. Identification of the 46 major and minor SCCmec variants currently known relies on detection of the presence or absence of at least 22 of 34 binary targets (Stephens et al., 2007). mPCR-based reverse line-blot hybridization assay (mPCR/RLB) can overcome the limitations of current methods by allowing rapid and simultaneous detection of a large number of different PCR products using membrane-bound sequence-specific probes (Kong \& Gilbert, 2006). This technique has been
Table 1. cont.

\begin{tabular}{|c|c|c|c|}
\hline ID & Strain & spa type* & Clonal type $\dagger$ \\
\hline 32 & PAH $1 末$ & t019 & ST30-SCCmec-IV \\
\hline 33 & IP01M2046‡ & $\mathrm{t} 1958$ & ST78-SCCmec-IV \\
\hline 34 & SJOG $30 \ddagger$ & $\mathrm{t} 127$ & ST1-SCCmec-IV \\
\hline 35 & RHH58 & T127 & $\mathrm{ST} \sim 1-\mathrm{SCC} m e c-\mathrm{IV}$ \\
\hline 36 & IMVS $67 \ddagger$ & t008 & ST8-SCCmec-V \\
\hline 37 & RPH 74‡ & $\mathrm{t} 123$ & ST45-SCCmec-V \\
\hline 38 & J710566‡ & t065 & ST45-SCCmec-V \\
\hline 39 & B827549‡ & $\mathrm{t} 1784$ & ST $\sim 1$-SCCmec-new \\
\hline 40 & SN39: & $\mathrm{t} 127$ & $\mathrm{ST} \sim 1$-SCCmec-new \\
\hline 41 & $\mathrm{RPH} 2 \ddagger$ & $\mathrm{t} 190$ & ST8-SCCmec-new \\
\hline 42 & C801535‡ & t325 & ST88-SCCmec-new \\
\hline
\end{tabular}

* spa type described by Cai et al. (2007).

$\dagger \mathrm{ST} \sim$, single nucleotide polymorphism (SNP) type as described previously using the computer program Minimum SNPs to compare with existing MLST data (Huygens et al., 2004, 2006). SCCmec, staphylococcal cassette chromosome. ST, multilocus sequence type (MLST). Information provided by strain donors.

¥Provided by Dr Philip Giffard, Cooperative Research Centre for Diagnostics, Queensland University of Technology, Brisbane, Australia, and Dr Graeme Nimmo, Queensland Health Pathology Services (QHPS), Princess Alexandra Hospital, Brisbane, Australia, and tested in previous studies (Huygens et al., 2004, 2006; Stephens et al., 2006; Coombs et al., 2004).

$\S$ Seven isolates, which had been used in previous studies (Oliveira et al., 2006b), were provided by Professor Herminia de Lencastre, Instituto de Tecnologia Química e Biológica, Universidade Nova de Lisboa, Oeiras, Portugal; spa types identified in this study were identical with those previously reported for these strains (Cai et al., 2007).

successfully applied to molecular typing of Streptococcus agalactiae (Kong et al., 2005), Streptococcus pneumoniae (Kong et al., 2006) and Staphylococcus aureus (Cai et al., 2007).

The aim of this study was to develop a new discriminatory method for MRSA SCCmec typing based on MPCR/RLB suitable for the rapid identification and classification of the MRSA isolates in a clinical laboratory.

\section{METHODS}

Bacterial isolates. Seven well-characterized reference strains [Cai et al., 2007; kindly provided by Professor Herminia de Lencastre, Instituto de Tecnologia Química e Biológica, Universidade Nova de Lisboa (UNL), Oeiras, Portugal] and 35 previously characterized clinical strains (provided by Drs Graeme Nimmo, Queensland Health Pathology Services, Princess Alexandra Hospital, Brisbane, Queensland, Australia, and Philip Giffard, Cooperative Research Centre for Diagnostics, Queensland University of Technology, Brisbane, Queensland, Australia) were used in this study (Table 1). Isolates were stored at $-70{ }^{\circ} \mathrm{C}$, until DNA extracts were prepared, as previously described (Cai et al., 2007); the latter were stored at $-20{ }^{\circ} \mathrm{C}$ until tested. 
Primer and probe design. The mPCR primers (Kondo et al., 2007) were modified to adjust $T_{\mathrm{m}}$ values to $60-65{ }^{\circ} \mathrm{C}$ and labelled with biotin. Immediate downstream or upstream sequences of the published sense and antisense primers were used to design antisense or sense $\mathrm{mPCR} / \mathrm{RLB}$ probes as described previously (Kong \& Gilbert, 2006). An inner primer was designed for each of the published sense and anti-sense primers with expected product size of around $100 \mathrm{bp}$. In total, 45 primer sets and 49 probes were assembled (some primer sets contained two probes). All primer sets were tested with each of the 42 reference strains using uniplex PCR (uPCR) and mPCR/RLB (Table 2).

UPCR and MPCR reactions. PCR amplifications were performed in a thermal cycler with HotStar Taq DNA polymerase (Qiagen). A $25 \mu \mathrm{l}$ uPCR mixture was prepared follows: $2.5 \mu \mathrm{l} 10 \times$ PCR buffer with a final $\mathrm{MgCl}_{2}$ concentration of $1.5 \mathrm{mM}, 0.2 \mathrm{mM}$ deoxynucleoside triphosphates, $12.5 \mu \mathrm{M}$ of each of the forward and reverse primers, $2 \mu \mathrm{l}$ template DNA, $0.2 \mu \mathrm{l}$ Qiagen Hotstar Taq polymerase $\left(5 \mathrm{U} \mathrm{\mu l}^{-1}\right.$ ) and molecular biology grade $\mathrm{H}_{2} \mathrm{O}$ (Eppendorf) was added to a total volume of $25 \mu \mathrm{l}$. Two mPCR mixes were prepared based on uPCR results for the 42 reference strains: the first contained 39 primer pairs (primer sets $1-18,21-24$ and 29-45, Table 2), which produced positive uPCR results for at least one strain tested, and the second contained six primer pairs (primer sets 19,20, and 25-28, Table 2) that were uPCR negative for all the strains tested, but showed in silico matches with one or more SCCmec sequences in GenBank.

Each mPCR mix comprised $2 \mu$ DNA template and $3 \mu \mathrm{l} 10 \times$ PCR buffer with a final concentration of: $1.5 \mathrm{mM} \mathrm{MgCl}_{2}$ (Qiagen), $0.2 \mathrm{mM}$ dNTP, $1.5 \mathrm{U}$ HotStar Taq DNA polymerase and $12.5 \mu \mathrm{M}$ of each of the forward and reverse primers. Molecular biology grade $\mathrm{H}_{2} \mathrm{O}$ (Eppendorf) was added to a total volume of $30 \mu \mathrm{l}$. PCR was performed according to the Qiagen HotStar Taq polymerase kit instructions: $95{ }^{\circ} \mathrm{C}$ for $15 \mathrm{~min}$; 35 cycles of $94{ }^{\circ} \mathrm{C}$ for $30 \mathrm{~s}, 55^{\circ} \mathrm{C}$ for $30 \mathrm{~s}, 72{ }^{\circ} \mathrm{C}$ for $45 \mathrm{~s}$, and $72{ }^{\circ} \mathrm{C}$ for $10 \mathrm{~min}$. PCR products were stained with SYBR safe DNA gel stain and visualized in a $2 \%$ agarose gel.

Reverse line blot (RLB) hybridization. The RLB hybridization assay was performed as described previously (Kong \& Gilbert, 2006; Cai et al., 2007). Briefly, all probes, synthesized with a $5^{\prime}$ amine modification at concentrations of $5-10 \mu \mathrm{M}$ in $0.5 \mathrm{M} \mathrm{NaHCO}_{3}$ $(\mathrm{pH} 8.4)$, were covalently linked to a Biodyne $\mathrm{C}$ hydrophilic nylon $6,6(0.45 \mu \mathrm{m})$ membrane (PALL Gelman Laboratory), which was negatively activated by incubation in freshly prepared $16 \%(\mathrm{w} / \mathrm{v}) 1$ ethyl-3-(3-dimethylaminopropyl)carbodiimide (EDAC) (Sigma). The membrane was placed in a miniblotter (MN45, Immunetics) on a support cushion. A volume of $20 \mu \mathrm{l}$ of PCR products was added to $150 \mu \mathrm{l} 2 \times$ SSPE (Amresco) in $0.1 \%$ SDS (Sigma), denatured at $100{ }^{\circ} \mathrm{C}$ for $10 \mathrm{~min}$ and cooled immediately on ice. Hybridization was conducted by adding $150 \mu \mathrm{l}$ of the diluted denatured PCR products to alternate slots on the miniblotter, which was then incubated at $60{ }^{\circ} \mathrm{C}$ for $60 \mathrm{~min}$. The membrane was washed twice in $2 \times$ SSPE buffer in $0.5 \%$ SDS at $60{ }^{\circ} \mathrm{C}$ for $10 \mathrm{~min}$, incubated in peroxidase-labelled streptavidin conjugate (Roche) at $42{ }^{\circ} \mathrm{C}$ for $60 \mathrm{~min}$, then washed twice in $2 \times$ SSPE buffer in $0.5 \%$ SDS at $42{ }^{\circ} \mathrm{C}$ for $10 \mathrm{~min}$ and twice in $2 \times$ SSPE buffer at room temperature for $5 \mathrm{~min}$. For detection, the membrane was incubated in $15 \mathrm{ml}$ chemiluminescence blotting substrate ECL detection reagent (Amersham, GE Healthcare) for 2 min, covered with transparent plastic sheet in a film cartridge and exposed with chemiluminescence film (Amersham Hyperfilm ECL, GE Healthcare) for $4 \mathrm{~min}$. RLB results were regarded as positive when a hybridization dot signal was clearly visible.

Quality control of mPCR/RLB results. To ensure reproducibility of $\mathrm{mPCR} / \mathrm{RLB}$ results, positive and negative controls were run on each membrane. The positive control was a mixture of DNA extracts from MRSA strains COL, BK2464, E822485, ANS46, DEN2988, PAH1, IP01M2046, RHH58, RPH74, B827549 and RPH2, which together contain all 43 probe targets; the negative control was master mix only, without DNA. Any signal produced by a test isolate that was similar to or stronger than the corresponding signal of the positive control was identified as positive. A signal produced by the negative control was assumed to be due to contamination and the assay was repeated. In practice, rare equivocal results would be resolved by UPCR for the relevant target, with sequencing of the amplicon, if required, as 'gold standard', but for the purpose of this study, all mPCR/RLB results were confirmed with uPCR.

\section{SCCmec gene and staphylococcal whole-genome sequence} analysis. Thirty-five SCCmec gene sequences published in NCBI GenBank, including 20 known SCCmec type (I-VI) and subtype sequences and 14 Staphylococcus aureus genome and 1 meticillinresistant $S$. epidermidis genome sequences were analysed as reference sequences and tested in silico using the mPCR/RLB assay. These sequences (with strain numbers; and DDBJ/EMBL/GenBank accession numbers) were as follows: (a) 18 SCCmec sequences: type I.1 SCCmec (NCTC10442; AB033763); type II.1 SCCmec (N315; D86934); type II.2 SCCmec (JCSC3063; AB127982); type II.B SCCmec (AR05; AJ810123); type II.3 (type IIE) SCCmec (AR13.1/ 3330.2; AJ810120); type II.4 SCCmec (RN7170; AB261975); type III.1 SCCmec (HU25; AF422696); type III.1 SCCmec and SCCmercury (85/ 2082; AB037671); type IV.1 SCCmec (CA05 [JCSC1968]; AB063172); type IV.2 SCCmec (JCSC1978 [8/6-3P] AB063173); type IV.3 SCCmec (81/108 [MR108]; AB096217); type IV.C SCCmec (2314; AY271717); type IV.4 SCCmec (JCSC4469; AB097677); type IV.E SCCmec (AR43/ 3330.1; AJ810121); type IV.g SCCmec (M03-68; DQ106887); type IV.h (PL72; AF411936); type V SCCmec (JCSC3624 [WIS]; AB121219); type VI SCCmec (HDE288; AF411935); and (b) $14 \mathrm{~S}$. aureus genomes: COL (CP000046), JH1 (CP000736), JH9 (CP000703), MRSA252 (BX571856), MW2 (BA000033), Mu3 (AP009324), Mu50 (BA000017), N315 (BA000018), USA300 (CP000255), USA300_TCH1516 (CP000730), RF122 (AJ938182), MSSA476 (BX571857), NCTC8325 (CP000253), Newman (AP009351).

In addition, the $\mathrm{mPCR} / \mathrm{RLB}$ system was tested in silico against wholegenome and partial SCCmec sequences of seven non-MRSA strains: five meticillin-susceptible S. aureus (MSSA) [AJ938182, BX571857, CP000253, AP009351, AM983545 (SSCmec type II)], plus one meticillin-resistant coagulase-negative Staphylococcus [S. epidermidis CP000029 (SCCmec type II)] and Staphylococcus pseudintermedius [AM904732 (SCCmec type III)].

GenBank SCCmec sequence searches and alignments and design of primers and probes were conducted using BioManager (Sydney Bioinformatics, https://www.angis.org.au/) and Sigma (http:// www.sigma-genosys.com/calc/DNACalc.asp) browsers. Results of BLASTN alignments of designed probe sequences with all currently available SCCmec types (I-VI) and subtype sequences (35 GenBank reference sequences) including whole-genome and partial SCCmec sequences of non-MRSA staphylococci were applied for in silico prediction of RLB hybridization patterns. The UPCR and MPCR/RLB results were recorded as binary data, exported into BioNumerics software (version 4.6, Applied Maths). Dendrograms were generated using the categorical coefficient and clustering by UPGMA algorithm.

Statistical analysis. The discriminatory power was estimated by calculating Simpson's index of diversity (Hunter \& Gaston, 1988) and the $95 \%$ confidence intervals (CI) were determined as described previously (Grundmann et al., 2001). 
Table 2. Primers and probes used in $\mathrm{mPCR} / \mathrm{RLB}$ for $\mathrm{SCC} m e c$ genotyping

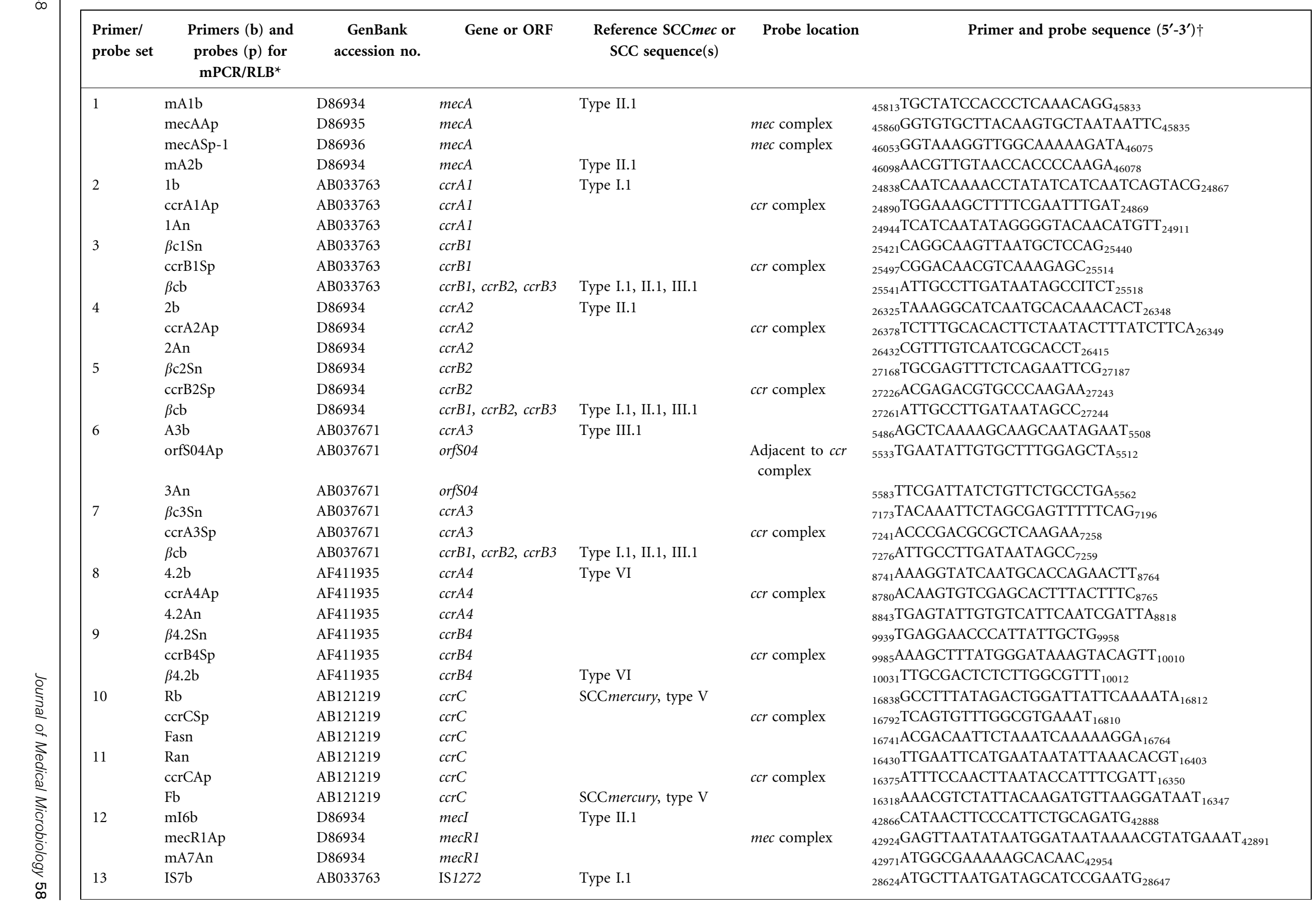


Table 2. cont

\begin{tabular}{|c|c|c|c|c|c|c|}
\hline $\begin{array}{l}\text { Primer/ } \\
\text { probe set }\end{array}$ & $\begin{array}{c}\text { Primers }(b) \text { and } \\
\text { probes }(p) \text { for } \\
\text { mPCR/RLB }{ }^{\star}\end{array}$ & $\begin{array}{c}\text { GenBank } \\
\text { accession no. }\end{array}$ & Gene or ORF & $\begin{array}{l}\text { Reference SCCmec or } \\
\text { SCC sequence(s) }\end{array}$ & Probe location & Primer and probe sequence $\left(5^{\prime}-3^{\prime}\right) \dagger$ \\
\hline \multirow{5}{*}{14} & orfS034Ap & $\mathrm{AB} 033763$ & orfS034 & & $\begin{array}{l}\text { Adjacent to mec } \\
\text { complex }\end{array}$ & 28670 TTTTGATTTTGTGATAGCCACA 28649 \\
\hline & mA7An-1 & $\mathrm{AB} 033763$ & orfS035 & & & ${ }_{28726}$ ATAAAGCATCTACTTTTGGATTTACTCT 28699 \\
\hline & IS7Sn & $\mathrm{AB} 033763$ & $\operatorname{mec} R 1$ & & & ${ }_{31288} \mathrm{CACCTTCTACACCTCCATATCA}_{31309}$ \\
\hline & mecASp-2 & AB033763 & $m e c A$ & & mec complex & 31395 AAAGATAAAAATTGTTCCACTTATTTTAATAG 31426 \\
\hline & $\mathrm{mA} 7 \mathrm{~b}$ & $\mathrm{AB} 033763$ & mecA & Type I.1, II.1, V & & ${ }_{31450}$ ATATACCAAACCCGACAACTACA $_{31428}$ \\
\hline \multirow[t]{3}{*}{15} & IS2(iS-2)Sn & AB121219 & IS 431 & & & ${ }_{8670}$ACAGATCAGGCACCTTCAAC $_{8689}$ \\
\hline & IS431Sp & AB121219 & IS431 & & $\begin{array}{l}\text { Adjacent to mec } \\
\text { complex }\end{array}$ & ${ }_{8721}$ TTTAAACTTAAACCTGACTGTCATTGT $_{8747}$ \\
\hline & IS2(iS-2)b & AB121219 & IS431 & Type V & & ${ }_{8772}$ TGAGGTTATTCAGATATTTCGATGT $_{8748}$ \\
\hline \multirow[t]{4}{*}{16} & $1 \mathrm{a} 3 \mathrm{~b}$ & AB033763 & E007 & Type I.1 & & ${ }_{5277}$ TTTTAGGAGGTAATCTCCTTGATG 5300 \\
\hline & E007Ap & AB033763 & E007 & & $\mathrm{J} 1$ & ${ }_{5328}$ TTTATAACATTGTCCTCTTTACCTTCAG ${ }_{5301}$ \\
\hline & E007Sp & $\mathrm{AB} 033763$ & E007 & & $\mathrm{J} 1$ & ${ }_{5387}$ AATAAGCATACATGGAGACGTTTT ${ }_{5410}$ \\
\hline & $1 \mathrm{a} 4 \mathrm{~b}$ & AB033763 & E007 & Type I.1 & & ${ }_{5431}$ TTTTGCGTTTGCATCTCTACC 5411 \\
\hline \multirow[t]{3}{*}{17} & $4 \mathrm{alb}$ & $\mathrm{AB} 063172$ & CQ002 & Type IV.1 & & ${ }_{4726}$ TTTGAATGCCCTCCATGAATAAAAT $_{4750}$ \\
\hline & CQ002Ap & AB063172 & CQ002 & & $\mathrm{J} 1$ & ${ }_{4777}$ CGGGTAATATGAGAATAAATAAAGAAG $_{4751}$ \\
\hline & $4 \mathrm{a} 1 \mathrm{An}$ & $\mathrm{AB} 063172$ & CQ002 & & & ${ }_{4844}$ GCGTATTATGATACTAAGCAAAGTATTAAAATAG $_{4811}$ \\
\hline \multirow[t]{3}{*}{18} & $4 \mathrm{a} 3 \mathrm{Sn}$ & AB063172 & CQ002 & & & ${ }_{5079}$ TTGAAATCCTGGGAATCTATCACT $_{5102}$ \\
\hline & CQ002Sp & AB063172 & CQ002 & & $\mathrm{J} 1$ & 5133 ACATGCTGTAGTCATTTTATACTGTTTG 5160 \\
\hline & $4 \mathrm{a} 3 \mathrm{~b}$ & AB063172 & CQ002 & Type IV.1 & & ${ }_{5183}$ AGCATATAGAAAAGATAGAAGTTCGAAAGA $_{5161}$ \\
\hline \multirow[t]{3}{*}{19} & $4 \mathrm{~b} 3 \mathrm{~b}$ & AB063173 & M001 & Type IV.2, II.3 & & ${ }_{2457}$ AACCAACAGTGGTTACAGCTT $_{2477}$ \\
\hline & M001Ap & AB063173 & M001 & & $\mathrm{J} 1$ & ${ }_{2509}$ AAATTTTAAACAATTCTAATTCAAATAAGTTG 2478 \\
\hline & $4 \mathrm{~b} 3 \mathrm{An}$ & AB063173 & M001 & & & ${ }_{2562}{ }^{A T C A A A T T T T T T A G C A G A T G T T G G ~} 2539$ \\
\hline \multirow[t]{3}{*}{20} & $4 \mathrm{~b} 4 \mathrm{Sn}$ & AB063173 & M001 & & & ${ }_{3041}$ATAGTGAACGTACTGATAACAAATTATTAGAT $_{3072}$ \\
\hline & M001Sp & $\mathrm{AB} 063173$ & M001 & & $\mathrm{J} 1$ & 3131 ATCATAAAATTACAGCTTACGATATACAAA 3160 \\
\hline & $4 \mathrm{~b} 4 \mathrm{~b}$ & АВ063173 & M001 & Type IV.2, II.3 & & ${ }_{3182}$ CGGATTTTAGACTCATCACCAT $_{3161}$ \\
\hline \multirow[t]{4}{*}{21} & $4 \mathrm{c} 4 \mathrm{~b}$ & AB096217 & CR008 & Type IV.3 & & ${ }_{8266}$ AAGAAAAGGAAATCGATGTCATTATAA 8240 \\
\hline & CR008Ap & AB096217 & CR008 & & $\mathrm{J} 1$ & ${ }_{8055}$ TCCTACTCCTATAAAGAGGTTACAGAATTATT $_{8024}$ \\
\hline & CR008Sp & AB096217 & CR008 & & $\mathrm{J} 1$ & ${ }_{8208}$ CAGATAGTATGGTACATATCCTTTAGGATT $_{8237}$ \\
\hline & $4 \mathrm{c} 5 \mathrm{~b}$ & AB096217 & CR008 & Type IV.3 & & ${ }_{7998}$ CTGCATCCATTTCTCAGGAGTTAG 8021 \\
\hline \multirow[t]{3}{*}{22} & $4 \mathrm{~d} 3 \mathrm{~b}$ & AB097677 & CD002 & Type IV.4 & & ${ }_{2390}$AATTCACCCGTACCTGAGAA $_{2409}$ \\
\hline & CD002Ap & AB097677 & CD002 & & $\mathrm{J} 1$ & 2440AGGATTTGGTGTATATAAAGGAGTGAAATA 2411 \\
\hline & $4 \mathrm{~d} 3 \mathrm{An}$ & AB097677 & CD002 & & & ${ }_{2496}{ }_{6}$ GCCTTTACACTAAGTACAGGGACATT 2471 \\
\hline \multirow[t]{3}{*}{23} & $4 \mathrm{~d} 4 \mathrm{Sn}$ & АВ097677 & CD002 & & & ${ }_{3497}$ TCTCTTAGAATTTTTTTGATATATTCATCTGA 3528 \\
\hline & CD002Sp & AB097677 & $\mathrm{CD} 002$ & & $\mathrm{~J} 1$ & 3575 TGTACATCTCGTATGTTAGAAACATTATTTAAA 3607 \\
\hline & $4 \mathrm{~d} 4 \mathrm{~b}$ & AB097677 & CD002 & Type IV.4 & & ${ }_{3635}$ GTCCAGAATGTGGTTATAAGATAGCTA 3609 \\
\hline \multirow[t]{3}{*}{24} & kdpB1b & D86934 & $k d p B$ & Type II.1 & & ${ }_{12439}{ }^{\text {TGAGATTACTTCAGAACCAGGTCAT }} 12415$ \\
\hline & kdpBAp & D86934 & $k d p B$ & & $\mathrm{~J} 1$ & ${ }_{12190}$ GGCTATAGGGATTGCAGGG $_{12172}$ \\
\hline & kdpBSp & D86934 & $k d p B$ & & $\mathrm{~J} 1$ & ${ }_{12388}$ CAAACCAATCATTTTATCTAAAAATG $_{12413}$ \\
\hline
\end{tabular}


Table 2. cont.

\begin{tabular}{|c|c|c|c|c|c|c|}
\hline $\begin{array}{l}\text { Primer/ } \\
\text { probe set }\end{array}$ & $\begin{array}{c}\text { Primers }(b) \text { and } \\
\text { probes }(p) \text { for } \\
\text { mPCR/RLB }\end{array}$ & $\begin{array}{c}\text { GenBank } \\
\text { accession no. }\end{array}$ & Gene or ORF & $\begin{array}{l}\text { Reference SCCmec or } \\
\text { SCC sequence(s) }\end{array}$ & Probe location & Primer and probe sequence $\left(5^{\prime}-3^{\prime}\right) \dagger$ \\
\hline \multirow{3}{*}{25} & kdpB2b & D86934 & $k d p B$ & Type II.1 & & ${ }_{12150}$ TAAACTGTGTCACACGATCCAT $_{12171}$ \\
\hline & $2 \mathrm{~b} 3 \mathrm{~b}$ & AB127982 & S01 & Type II.2 & & ${ }_{1497}$ GCTCTAAAAGTTGGATATGCG 1517 \\
\hline & S01Ap & AB127982 & S01 & & $\mathrm{J} 1$ & ${ }_{1546}$ AAAGTAATCTATATGATCCCATTTTTGA 1519 \\
\hline \multirow{3}{*}{26} & $2 \mathrm{~b} 3 \mathrm{An}$ & $\mathrm{AB} 127982$ & S01 & & & ${ }_{1599}$ TTAGTATTTGTTACAATGCCCACA 1576 \\
\hline & $2 \mathrm{~b} 4 \mathrm{Sn}$ & AB127982 & S01 & & & ${ }_{2906}$ ACAGTTAGAACATGTTAATAAAAACATCG 2934 \\
\hline & S01Sp & AB127982 & S01 & & $\mathrm{J} 1$ & ${ }_{2963}$ CAAATTAAGCAAACTTACTGAATATTTAGC ${ }_{2992}$ \\
\hline \multirow{3}{*}{27} & $2 \mathrm{~b} 4 \mathrm{~b}$ & AB127982 & S01 & Type II.2 & & ${ }_{3014}$TGGATTGAATCGACTAGAATCG $_{2993}$ \\
\hline & II $4-3 b$ & AB261975 & RN06 & Type II.4 & & ${ }_{11846}$ AAGTACCGCTGAATATTGATAGTGAT ${ }_{11871}$ \\
\hline & RN06Ap & AB261975 & RN06 & & $\mathrm{J} 1$ & ${ }_{11909}$ CCTATTAATTGAGATATTGTATGATAATATCCTT $_{11876}$ \\
\hline \multirow{3}{*}{28} & II $4-3 \mathrm{An}$ & AB261975 & RN06 & & & ${ }_{11975}$ GGTTTTGTTTTTTCAATATAATACCTTG $_{11948}$ \\
\hline & II4-1Sn & AB261975 & RN06 & & & ${ }_{13715}$ TTGAAATACATGTTTTTGAAGAATATATATTG $_{13746}$ \\
\hline & RN06Sp & AB261975 & RN06 & & $\mathrm{J} 1$ & ${ }_{13797}$AGAAATTGATTTAATAAGTTCTTCTGAAAATA $_{13828}$ \\
\hline \multirow{3}{*}{29} & $\mathrm{II} 4-1 \mathrm{~b}$ & AB261975 & RN06 & Type II.4 & & ${ }_{13850}$ TTTCTACTCTAATCCTAATCACCGAAC $_{13829}$ \\
\hline & $3 a 1 b$ & $\mathrm{AB} 037671$ & Z004 & Type III.1 & & 3333 ATGGCTTCAGCATCAATGAG 3352 \\
\hline & Z004Ap & AB037671 & Z004 & & $\mathrm{J} 1$ & ${ }_{3381}$ AGTGCATTGCTTAATGATTTAATGTACT $_{3354}$ \\
\hline \multirow{3}{*}{30} & $3 \mathrm{a} 1 \mathrm{An}$ & AB037671 & Z004 & & & 3430 GACACCCGTATACAACGATGAC $_{3409}$ \\
\hline & $3 a 3 S n$ & AB037671 & Z004 & & & ${ }_{3734}$ACTGCAGCGGCTTTAGGA $_{3751}$ \\
\hline & Z004Sp & AB037671 & Z004 & & $\mathrm{J} 1$ & ${ }_{3793}$ GTTTAGATCATGGAATGGCACT 3814 \\
\hline \multirow{3}{*}{31} & $3 a 2 b$ & AB037671 & Z004 & Type III.1 & & ${ }_{3835}$ ATATCCTTCAAGCGCGTTTC 3816 \\
\hline & $5 \mathrm{a} 1 \mathrm{~b}$ & AB121219 & V024 & Type V & & ${ }_{26564}$ ACCTACAGCCATTGCATTATG $_{26584}$ \\
\hline & V024Ap & AB121219 & V024 & & $\mathrm{J} 1$ & ${ }_{26621}$ TCTTGATACATTTTAATATACTCACTAGTATTCGAT 26586 \\
\hline \multirow{3}{*}{32} & $5 \mathrm{a} 1 \mathrm{An}$ & AB121219 & V024 & & & 26684 GCGAATATAAATTCATATACTTTTTCATCA 26655 \\
\hline & $5 \mathrm{a} 2 \mathrm{Sn}$ & AB121219 & V024 & & & ${ }_{27617}$ GGGATTTCATCATCTTTTTAATGA 27640 \\
\hline & V024Sp & AB121219 & V024 & & $\mathrm{J} 1$ & 27671 AATTGATACTTATAAAATGTTAATAGCGCT ${ }_{27700}$ \\
\hline \multirow{3}{*}{33} & $5 \mathrm{a} 2 \mathrm{~b}$ & $\mathrm{AB} 121219$ & V024 & Type V & & ${ }_{27722}$ TGTATACATTTCGCCACTAGCT $_{27701}$ \\
\hline & ermAlb & D86934 & ermA & Type II.1 & & 35071AATTTAGTGAAACAATTTGTAACTATTGA 35099 \\
\hline & ermAAp & D86934 & ermA & & $\mathrm{J} 2$ & ${ }_{35126}$ TATCGAAGGAACAATTTCTTTCTATT $_{35101}$ \\
\hline \multirow{3}{*}{34} & ermA1An & D86934 & ermA & & & 35182 TAAAAACCAATTCCGACAGG 35163 \\
\hline & $\mathrm{mN} 5 \mathrm{Sn}$ & D86934 & $\mathrm{CN} 031$ & & & ${ }_{37724}$ CCCTCCCATATATATAACAAGTTTAGG $_{37750}$ \\
\hline & $\mathrm{CN} 031 \mathrm{Sp}$ & D86934 & $\mathrm{CN} 031$ & & $\mathrm{~J} 2$ & ${ }_{37783}$ ATCCACTAAGTGTACTGAAAAATACTTG $_{37810}$ \\
\hline \multirow{3}{*}{35} & $\mathrm{mN} 5 \mathrm{~b}$ & D86934 & $\mathrm{CN} 031$ & & & ${ }_{37833}$TTGCTTCGGGACTTACCTCTAGT $_{37811}$ \\
\hline & $\mathrm{cad} 4 \mathrm{~b}$ & AB037671 & $c a d B$ & Type III.1 & & ${ }_{16107}$ ATTGCGATTCTTTCCGATATGG 16128 \\
\hline & cadBAp & AB037671 & $\operatorname{cadB}$ & & $\mathrm{J} 2$ & ${ }_{16156}{ }^{C T A T T T A A T G C T A C C A A A A T A G T G G C T C ~}{ }_{16129}$ \\
\hline \multirow{3}{*}{36} & cad4An & AB037671 & Z020-Z021 spacer & & & ${ }_{16213}$ TTCCGTAAATTGCACACATATAGTACTT $_{16186}$ \\
\hline & $\mathrm{mN} 5 \mathrm{Sn}$ & AB037671 & Z023 & & & ${ }_{17537}$ CCCTCCCATATATATAACAAGTTTAGGA $_{17564}$ \\
\hline & Z023Sp & AB037671 & Z023 & & $\mathrm{J} 2$ & ${ }_{17594}$TGATCCACTAAGTGTACTGAAAAATACTTG $_{17623}$ \\
\hline \multirow{3}{*}{37} & $\mathrm{mN} 5 \mathrm{~b}$ & AB037671 & CN030, CZ021 & Type II.1, III.1 & & ${ }_{17646}{ }^{\text {TTGCTTCGGGACTTACCTCTAGT }}{ }_{17624}$ \\
\hline & $\mathrm{mAlb}$ & D86934 & mecA & Type II.1, III.1 & & ${ }_{45813}$TGCTATCCACCCTCAAACAGG $_{45833}$ \\
\hline & antAp & D86934 & mecA & & $\mathrm{J} 3$ & ${ }_{45859}$ GTGTGCTTACAAGTGCTAATAATTCA $_{45834}$ \\
\hline
\end{tabular}


Table 2. cont.

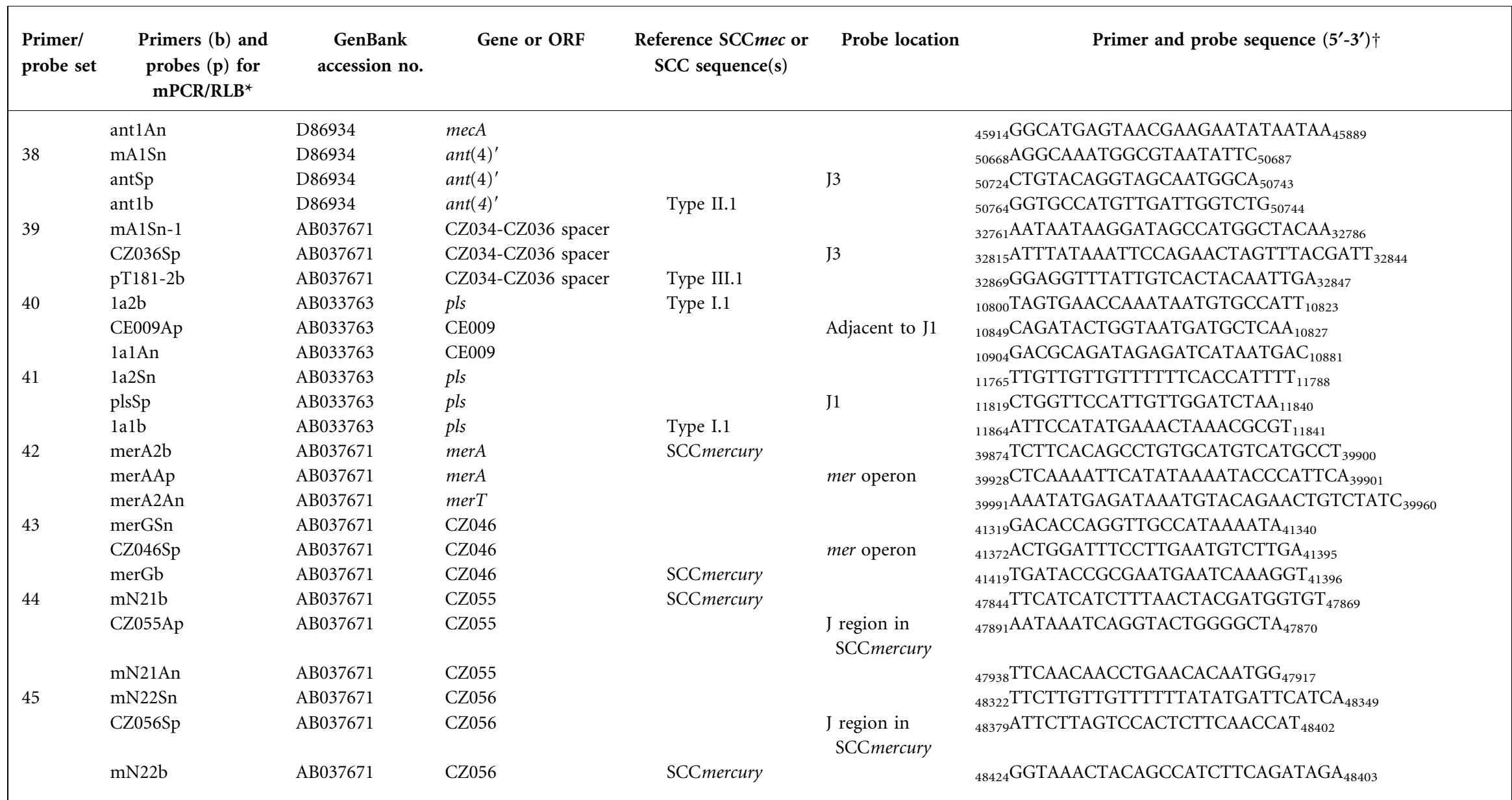

${ }^{*}$ S, sense; A, antisense; b, biotin-labelled primers (at the $5^{\prime}$ end); n, non-biotin-labelled primers; $\mathrm{p}$, $\mathrm{C}^{6}$-amine-labelled-probes (at the $5^{\prime}$ end). All primers labelled with biotin were based on previously published sequences (Kondo et al., 2007). Non-biotin-labelled primers and probes were designed for this study.

$\dagger$ Numbers indicate sequence position in relevant GenBank sequences. 


\section{RESULTS AND DISCUSSION}

\section{SCCmec type characteristics of the reference strains in $\mathrm{mPCR} / \mathrm{RLB}$ assays}

All 42 reference strains were tested by $\mathrm{UPCR}$ and $\mathrm{mPCR}$ using all 45 primer sets (Table 2 ). The majority produced amplicons of the expected size in the uPCR, but six (primer sets 19, 20,25-28) gave negative results. The two groups of primers (the 39 that generated and six that did not generate amplicon signals in the uPCR) were further studied in two separate mPCRs. Fig. 1 shows mPCR/RLB findings using the 39 primers that had given positive results in uPCR. As the other six primer sets did not produce signals with the 42 test isolates, they were removed from the membrane, to save space, and labelled onto a second membrane for use in subsequent studies.
The uPCR results were consistent with those of mPCR/ $\mathrm{RLB}$, with the following exceptions: primers $\beta \mathrm{c} 1 \mathrm{Sn}$ and $\beta \mathrm{cb}$ amplified SCCmec from strains FH53, SJOG30, RHH58, B827549 and SN39 in the uPCR but produced no signal in mPCR/RLB with probe ccrB1Sp (Fig. 1, lanes 1, 34, 35, 39 and 40 , respectively), presumably due to a major sequence variation in the probe region. There were minor differences in intensities of signal dots but, since this is a binary typing system, clear signals similar to or stronger than positive controls on the membrane were regarded as positive. This approach was validated internally by the use of paired (sense/antisense) probes and by comparison of the signals within and between the probes. In our experience, a weak signal reflects minor sequence variation (1-2 bp) and an absent signal represents at least a 2 bp sequence change in the corresponding probe region, as suggested previously (Wang et al., 2008). However, discordant signals between

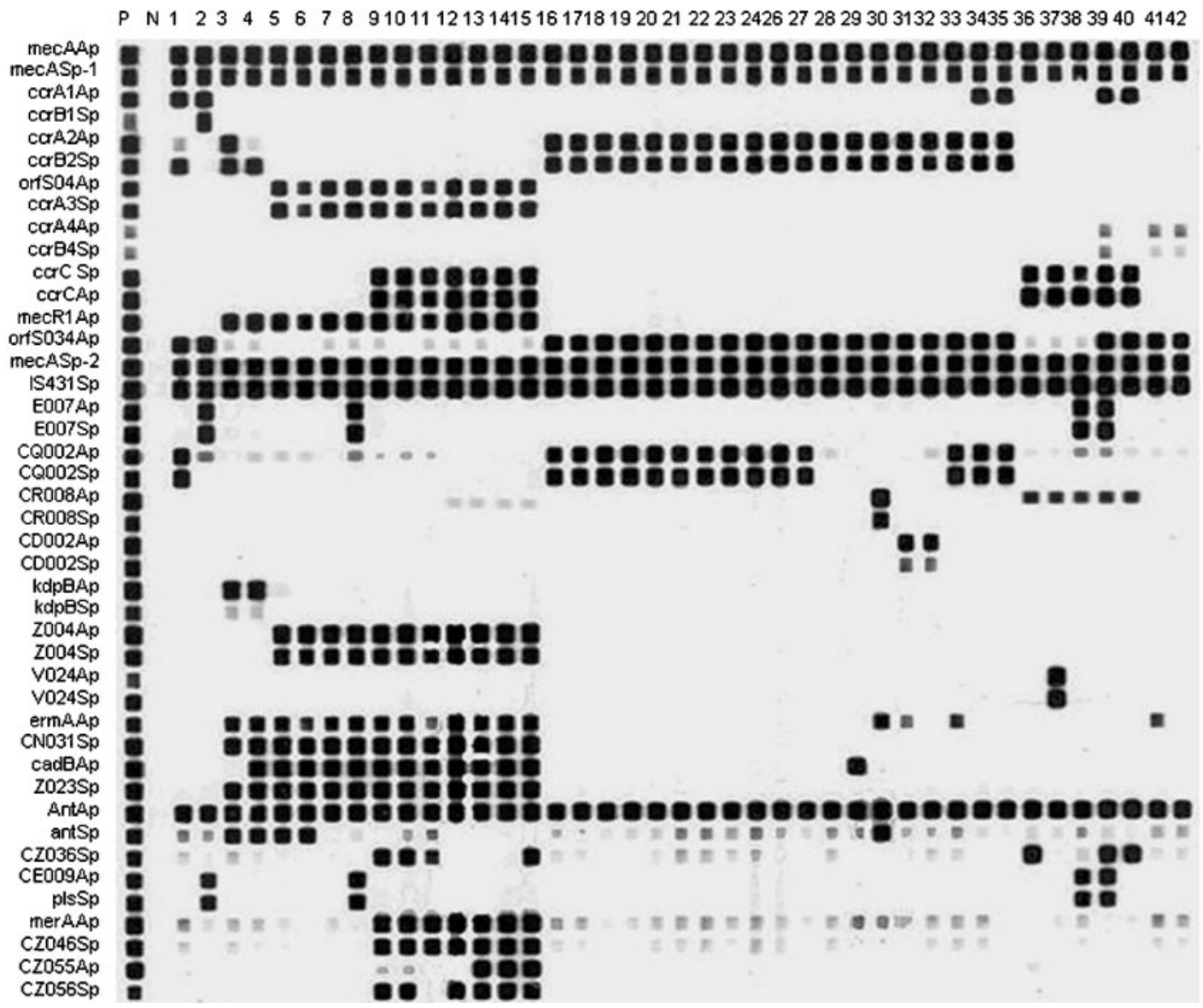

Fig. 1. RLB patterns of 41 reference strains of MRSA. Lane $P$, artificial positive control, Lane $N$, negative control (PCR master mix) Lanes 1-42: 1, FH53; 2; COL; 3, BK2464; 4, E822485; 5, RPAH18; 6, RPAH15; 7, HDG2; 8, 14176-5710; 9, RDH81; 10, AH1; 11, AH13; 12, HU25; 13, K704540; 14, K711532; 15, ANS46; 16, PC8; 17, FH43; 18, RPH85; 19, RHH10; 20, B8-10; 21, PAH58; 22, E804531; 23, CH97; 24, RBH98; 26, IP01M1081; 27, MW2; 28, CH16; 29, CH69; 30, DEN2988; 31, F829549; 32, PAH1, 33, IP01M2046; 34, SJOG30; 35, RHH58; 36, IMVS67; 37, RPH74; 38, J710566; 39, B827549; 40, SN39; 41, RPH2; 42, C801535. [Note: strain 25 (13792-4492) was not included; it gave results identical to those of strain 24 (RBH98).] 
probe pairs (one positive - weak or strong - and one negative) can occur but are rare. uPCR and sometimes sequencing, using the same primers as used for that target in the mPCR, can be used to determine whether the discrepancy is due to a modified target (amplicon present) or a non-specific cross-reaction (amplicon absent).

\section{In silico comparison of probe sequences with SCCmec gene sequences}

The potential sensitivity of the probes designed in the study was assessed in an in silico experiment. The GenBank sequences of $14 \mathrm{~S}$. aureus whole-genome sequences, 14 partial SCCmec sequences and seven non-MRSA genome and SCCmec sequences were downloaded and compared with probe sequences from this study using BLASTN pairwise alignments. The results are expressed as virtual RLB profiles in Fig. 2, in which the six probes that gave negative results for the 42 reference strains were also included. This comparative analysis demonstrated that each of the 45 probes found homologous matches with at least one GenBank MRSA SCCmec sequence. It suggested that the six additional probes/primer sets could be potentially useful for SCCmec typing and should be retained in the $\mathrm{mPCR} / \mathrm{RLB}$.

It should be noted that sequences of MRSA SCCmec type II 2 (lane 3), type IIB (lane 4), type II.4 (lane 6, accession no. AB261975), type III.1 (lane 7), type IV.4 (lane 13, accession no. AB097677), and type VI (lane 18, accession no. AF411935) were deposited in GenBank as partial-length sequences and may represent only the subtype-definitive regions of the SCCmec gene (Kondo et al., 2007). The SCCmec type patterns identified by RLB in our 42 reference strains were generally consistent with those extrapolated from MRSA SCCmec gene sequences in the public domain. For example, the majority of SCCmec type IV strains (Fig. 1, lanes 15-26) had SCCmec profiles indistinguishable from that of the genome sequence ID 27 (Fig. 2, lane 27, CP000255). Altogether 15 of 42 strains $(35 \%)$ had the same RLB patterns as those of corresponding GenBank sequences. However, there was some heterogeneity in the RLB patterns of other reference strains compared with GenBank sequences of the same SCCmec types. For example, RLB patterns of reference strains of SCCmec type III differed from the published MRSA SCCmec type III subtype 1 sequence (Fig. 2, lane 8, AB037671). This heterogeneity suggests the existence of distinct SCCmec type III subtypes (Fig. 2).

Theoretical RLB profiles of seven non-MRSA genome sequences are shown in Fig. 2. Four of five MSSA demonstrated no matches between probes and genomes, but one MSSA, and both meticillin-resistant coagulasenegative Staphylococcus strains, contained some SCCmec elements, as shown by matching of variable numbers of probes with their genomic sequences. These results are consistent with previous findings of SCCmec elements in MSSA and coagulase-negative staphylococci (Mombach
Pinheiro Machado et al., 2007; Hanssen \& Sollid, 2007). It has been postulated that SCCmec elements can be transferred from coagulase-negative staphylococci to $S$. aureus (Shore et al., 2008). Our results suggest that our mPCR/RLB typing system could also be extended to study of the distribution of SCCmec elements in other staphylococci.

\section{Discrimination of SCCmec genotypes by mPCR/ RLB}

The mPCR/RLB SCCmec typing system developed in this study was based on an mPCR typing scheme described by Kondo et al. (2007) using $c c r$ and mec gene complexes types with further discrimination based on differences in junkyard region 1 and the presence or absence of integrated transposons and plasmids. Another recently developed SCCmec typing system used minimal subsets of targets on $\mathrm{ccr}$ and mec gene complexes and three junkyard regions to provide maximal discriminatory capacity (Stephens et al., 2007). The rationales for these two strategies are different: the former is based on known SCCmec variants, whereas the latter maximizes discrimination, by recognizing single nucleotide polymorphisms (SNPs) and is not limited to known SCCmec types. Our system was designed as an extension of the Kondo scheme, to discriminate major known SCCmec types and subtypes rapidly. However, it can be easily modified to accommodate probes for newly identified SCCmec elements.

The set of 42 well-characterized MRSA reference strains included isolates representing 5 SCCmec types, 9 clonal clusters, 22 spa types, 7 nuc types, 9 femA types, 14 toxin types and 15 MLST types (Cai et al., 2007). The mPCR/ RLB assay classified reference strains into 26 SCCmec subtypes, including one of SCCmec type I (1 strain), two of SCCmec II (2 strains), eight of SCCmec III (11 strains), eight of SCCmec IV (21 strains) and three of SCCmec V (3 strains). MRSA SCCmec type IV and its variants have increased in importance in recent years because they have been identified in virulent community-acquired strains (Deurenberg \& Stobberingh, 2008). Four of the previously unclassified strains demonstrated unique RLB patterns resembling SCCmec types IV and V (Fig. 3).

Different sequence-based typing methods targeting different types of gene may not be directly comparable but they are often proposed as alternatives to pulsed-field gel electrophoresis for strain typing. The mPCR/RLB SCCmec typing method showed similar discriminatory power to that of MLST and spa typing in this small but diverse strain collection, as indicated by Simpson indices of diversity of 0.916 (CI 88.1-95.1\%) for mPCR/RLB typing, 0.882 (CI 85.2-91.2\%) for MLST and 0.926 (CI 90.3$94.9 \%$ ) for spa typing, respectively. Further investigation is required to determine its practical utility for SCCmec typing of a larger collection of clinical isolates. However, combined with GenBank SCCmec sequence data, this typing scheme also has the potential to identify novel 


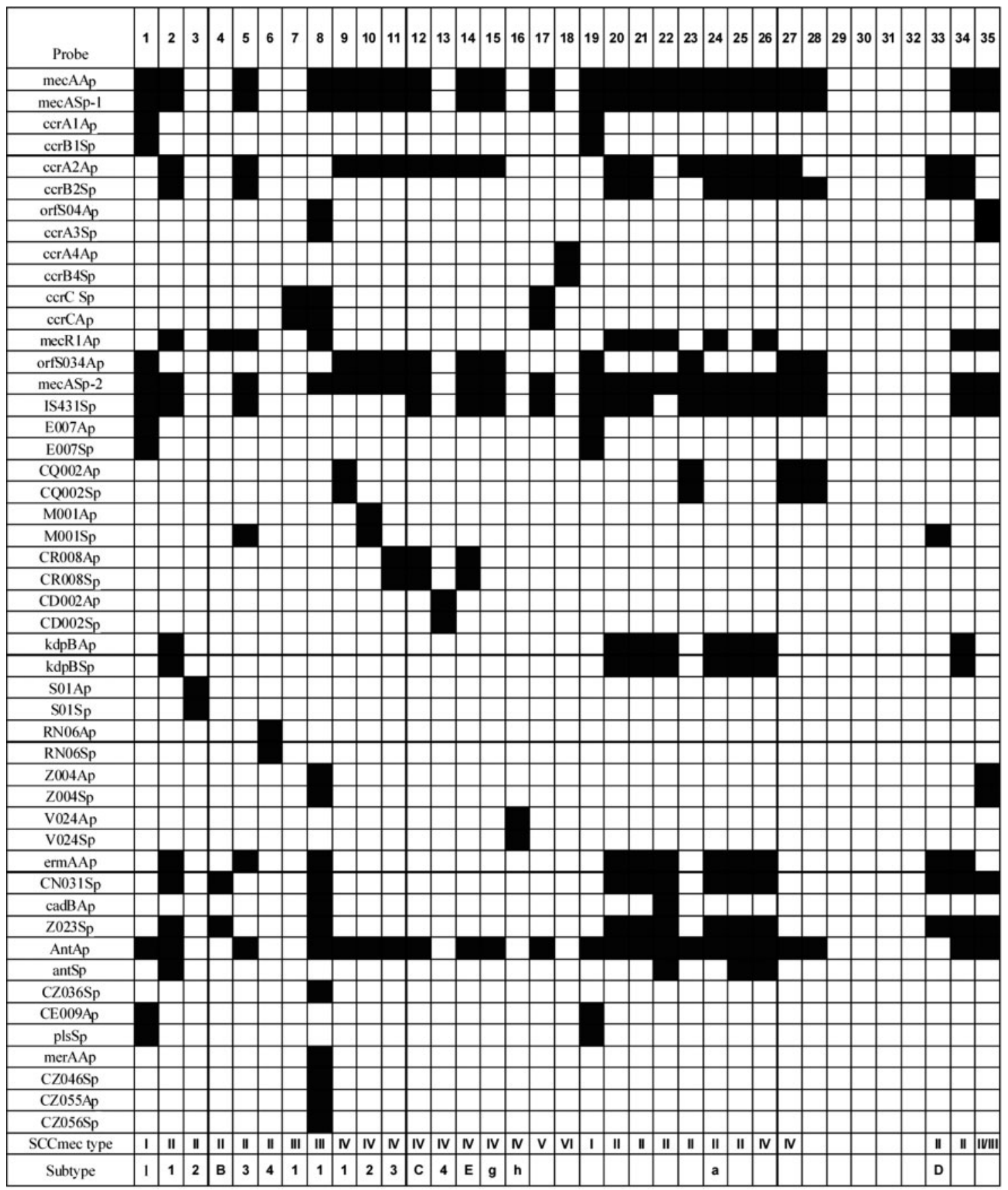

Fig. 2. Simulated in silico RLB results based on BLASTN search of RLB probes against SCCmec and whole-genome sequences of MRSA and seven non-MRSA staphylococcal genome sequences published in GenBank. Lanes 1-28 (28 MRSA sequences): 1, AB033763 (I.1); 2, D86934 (II.1); 3, AB127982 (II.2); 4, AJ810123 (IIB); 5, AJ810120 (II.3); 6, AB261975 (II.4); 7, AF422696 (III.1); 8, AB037671 (III.1); 9, AB063172 (IV.1); 10, AB063173 (IV.2); 11, AB096217 (IV.3); 12, AY271717 (IVC); 13 , AB097677 (IV.4); 14, AJ810121 (IVE); 15, DQ106887 (IVg); 16, AF411936 (IVh); 17, AB121219 (V); 18, AF411935 (VI); 19 , CP000046 (I); 20, CP000736 (II); 21, CP000703 (II); 22, BX571856 (II); 23, BA000033 (II); 24, AP009324 (Ila); 25 , BA000017 (II); 26, BA000018 (IV); 27, CP000255 (IV); 28, CP000730. Lanes 29-33 (5 MSSA sequences): 29, AJ938182; 30, BX571857; 31, CP000253; 32, AP009351; 33, AM983545 (IID). Lanes 34-35: 34, CP000029 (II) - meticillin-resistant S. epidermidis genome; 35, AM904732 (II/III) - meticillin-resistant S. pseudintermedius. The black squares represent perfect matches of probe sequences with the GenBank SCCmec sequences; the white squares represent probes for which there was no matching GenBank sequence at the time of study (July 2008). 


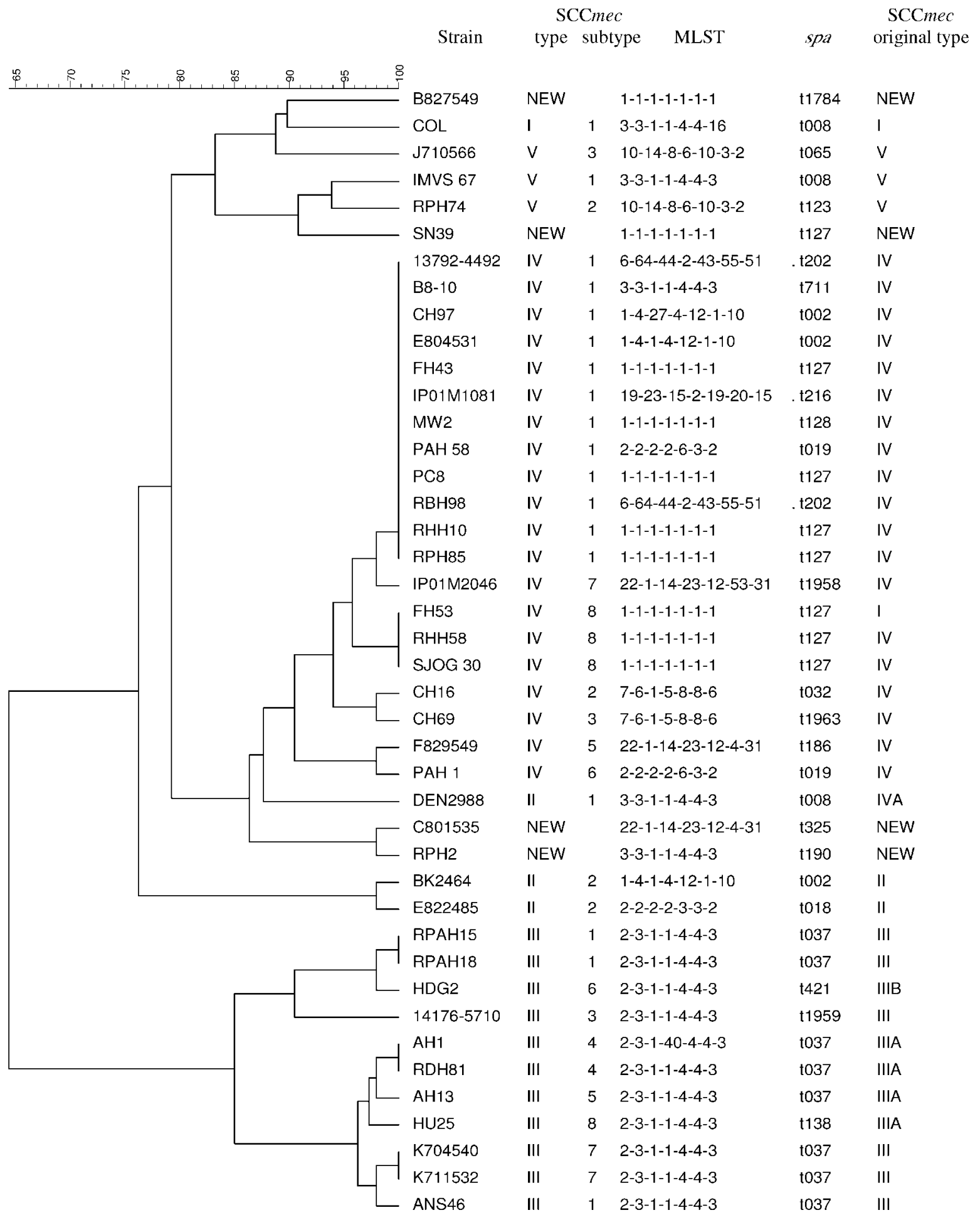

Fig. 3. Relationships between 42 MRSA strains determined by mPCR/RLB and other typing methods. The MLST and spa typing results were reported by Cai et al. (2007).

SCCmec types/subtypes among clinical MRSA isolates with increased accuracy and study relationships between them. Reference strain FH53 (lane 1, Fig. 2) was previously reported to be SCCmec type I (Cai et al., 2007). However, it appears to be similar to SCCmec IV reference and clinical strains as well as to the published SCCmec type IV sequence 
(Fig. 3). There was also one genetic marker difference (probe $\beta \mathrm{c} 2 \mathrm{Sp}$ ) for strain MW2 between the mPCR/RLB assay and in silico results which could be attributed to the polymorphism of the binding site.

\section{Advantages of the mPCR/RLB approach}

The SCCmec gene typing initially proposed by Kondo et al. (2007) involves several mPCRs that produce amplicons of significant size (up to $15 \mathrm{~kb}$ ) and requires gel-based detection. These features reduce the relevance of the typing scheme for infection-control-based interventions and tracking of introduction or transmission of different SCCmec types/subtypes. In contrast, the application of $\mathrm{mPCR} / \mathrm{RLB}$ to the SCCmec typing described here enables the simultaneous screening of up to 43 samples using two mPCRs with a turnaround time (including culturing of isolates, DNA extraction, mPCR setup and running, and RLB hybridization) of about two working days. The preparation of the RLB membrane takes less than $2 \mathrm{~h}$, and it can be reused at least 20 times based on our experience. The consumables cost is $\sim \mathrm{AU} \$ 8$ per isolate. The results of $\mathrm{mPCR} / \mathrm{RLB}$ can be represented as binary data, which are easily transferred and shared between diagnostic and public health laboratories, making the SCCmec typing easier to harmonize and suitable for rapid epidemiological studies of large numbers of isolates.

In conclusion, this SSCmec typing system improves our capacity to monitor molecular evolution and spread of MRSA and contributes to the development of effective strategies to control its dissemination. The application of the mPCR/RLB hybridization assay to MRSA SCCmec typing enhances the specificity, discriminatory power and throughput of the typing procedure. The sequence detection of up to $43 \mathrm{mPCR}$ products in a single hybridization assay can transform MRSA SCCmec typing from epidemiological library typing into a tool for nearreal-time infection control surveillance and MRSA hospital transmission tracking.

\section{ACKNOWLEDGEMENTS}

The authors thank Professor Herminia de Lencastre and Drs Philip Giffard and Graeme Nimmo for kindly sharing MRSA isolates for the study.

\section{REFERENCES}

Aires de Sousa, M. \& de Lencastre, H. (2004). Bridges from hospitals to the laboratory: genetic portraits of methicillin-resistant Staphylococcus aureus clones. FEMS Immunol Med Microbiol 40, 101-111.

Cai, Y., Kong, F., Wang, Q., Tong, Z., Sintchenko, V., Zeng, X. \& Gilbert, G. L. (2007). Comparison of single- and multilocus sequence typing and toxin gene profiling for characterization of methicillinresistant Staphylococcus aureus. J Clin Microbiol 45, 3302-3308.

Chongtrakool, P., Ito, T., Ma, X. X., Kondo, Y., Trakulsomboon, S., Tiensasitorn, C., Jamklang, M., Chavalit, T., Song, J. H. \& Hiramatsu, K.
(2006). Staphylococcal cassette chromosome mec (SCCmec) typing of methicillin-resistant Staphylococcus aureus strains isolated in 11 Asian countries: a proposal for a new nomenclature for SCCmec elements. Antimicrob Agents Chemother 50, 1001-1012.

Coombs, G. W., Nimmo, G. R., Bell, J. M., Huygens, F., O'Brien, F. G., Malkowski, M. J., Pearson, J. C., Stephens, A. J. \& Giffard, P. M. (2004). Genetic diversity among community methicillin-resistant Staphylococcus aureus strains causing outpatient infections in Australia. J Clin Microbiol 42, 4735-4743.

Daum, R. S., Ito, T., Hiramatsu, K., Hussain, F., Mongkolrattanothai, K., Jamklang, M. \& Boyle-Vavra, S. (2002). A novel methicillin-resistance cassette in community-acquired methicillin-resistant Staphylococcus aureus isolates of diverse genetic backgrounds. J Infect Dis 186, 13441347.

Deurenberg, R. H. \& Stobberingh, E. E. (2008). The evolution of Staphylococcus aureus. Infect Genet Evol 8, 747-763.

Grundmann, H., Hori, S. \& Tanner, G. (2001). Determining confidence intervals when measuring genetic diversity and the discriminatory abilities of typing methods for microorganisms. J Clin Microbiol 39, 4190-4192.

Hanssen, A. M. \& Sollid, J. U. (2007). Multiple staphylococcal cassette chromosomes and allelic variants of cassette chromosome recombinases in Staphylococcus aureus and coagulase-negative staphylococci from Norway. Antimicrob Agents Chemother 51, 1671-1677.

Hunter, P. R. \& Gaston, M. A. (1988). Numerical index of the discriminatory ability of typing systems: an application of Simpson's index of diversity. J Clin Microbiol 26, 2465-2466.

Huygens, F., Stephens, A. J., Nimmo, G. R. \& Giffard, P. M. (2004). mecA locus diversity in methicillin-resistant Staphylococcus aureus isolates in Brisbane, Australia, and the development of a novel diagnostic procedure for the Western Samoan phage pattern clone. J Clin Microbiol 42, 1947-1955.

Huygens, F., Inman-Bamber, J., Nimmo, G. R., Munckhof, W., Schooneveldt, J., Harrison, B., McMahon, J. A. \& Giffard, P. M. (2006). Staphylococcus aureus genotyping using novel real-time PCR formats. J Clin Microbiol 44, 3712-3719.

Ito, T. \& Hiramatsu, K. (1998). Acquisition of methicillin resistance and progression of multiantibiotic resistance in methicillin-resistant Staphylococcus aureus. Yonsei Med J 39, 526-533.

Ito, T., Katayama, Y. \& Hiramatsu, K. (1999). Cloning and nucleotide sequence determination of the entire mec DNA of pre-methicillinresistant Staphylococcus aureus N315. Antimicrob Agents Chemother 43, 1449-1458.

Ito, T., Katayama, Y., Asada, K., Mori, N., Tsutsumimoto, K., Tiensasitorn, C. \& Hiramatsu, K. (2001). Structural comparison of three types of staphylococcal cassette chromosome mec integrated in the chromosome in methicillin-resistant Staphylococcus aureus. Antimicrob Agents Chemother 45, 1323-1336.

Ito, T., Ma, X. X., Takeuchi, F., Okuma, K., Yuzawa, H. \& Hiramatsu, K. (2004). Novel type V staphylococcal cassette chromosome mec driven by a novel cassette chromosome recombinase, ccrC. Antimicrob Agents Chemother 48, 2637-2651.

Kondo, Y., Ito, T., Ma, X. X., Watanabe, S., Kreiswirth, B. N., Etienne, J. \& Hiramatsu, K. (2007). Combination of multiplex PCRs for staphylococcal cassette chromosome mec type assignment: rapid identification system for $m e c, c c r$, and major differences in junkyard regions. Antimicrob Agents Chemother 51, 264-274.

Kong, F. \& Gilbert, G. L. (2006). Multiplex PCR-based reverse line blot hybridization assay (mPCR/RLB) - a practical epidemiological and diagnostic tool. Nat Protoc 1, 2668-2680. 
Kong, F., Ma, L. \& Gilbert, G. L. (2005). Simultaneous detection and serotype identification of Streptococcus agalactiae using multiplex PCR and reverse line blot hybridization. J Med Microbiol 54, 1133-1138.

Kong, F., Brown, M., Sabananthan, A., Zeng, X. \& Gilbert, G. L. (2006). Multiplex PCR-based reverse line blot hybridization assay to identify 23 Streptococcus pneumoniae polysaccharide vaccine serotypes. J Clin Microbiol 44, 1887-1891.

Lalani, T., Federsoiel, J. J., Boucher, H. W., Rude, T. H., Bae, I.-G., Rybak, M. J., Tonthat, G. T., Corey, G. R., Stryjewski, M. E. \& other authors (2008). Associations between the genotypes of Staphylococcus aureus bloodstream isolates and clinical characteristics and outcomes of bacteremic patients. J Clin Microbiol 46, 2890-2896.

Ma, X. X., Ito, T., Tiensasitorn, C., Jamklang, M., Chongtrakool, P., Boyle-Vavra, S., Daum, R. S. \& Hiramatsu, K. (2002). Novel type of staphylococcal cassette chromosome mec identified in communityacquired methicillin-resistant Staphylococcus aureus strains. Antimicrob Agents Chemother 46, 1147-1152.

Milheirico, C., Oliveira, D. C. \& de Lencastre, H. (2007). Update to the multiplex PCR strategy for assigning of mec element types in Staphylococcus aureus. Antimicrob Agents Chemother 51, 3374-3377.

Mombach Pinheiro Machado, A. B., Reiter, K. C., Paiva, R. M. \& Barth, A. L. (2007). Distribution of staphylococcal cassette chromosome mec (SCCmec) types I, II, III and IV in coagulase-negative staphylococci from patients attending a tertiary hospital in southern Brazil. J Med Microbiol 56, 1328-1333.

Oliveira, D. C. \& de Lencastre, H. (2002). Multiplex PCR strategy for rapid identification of structural types and variants of the mec element in methicillin-resistant Staphylococcus aureus. Antimicrob Agents Chemother 46, 2155-2161.

Oliveira, D. C., Milheirico, C. \& de Lencastre, H. (2006a). Redefining a structural variant of staphylococcal cassette chromosome mec, SCCmec type VI. Antimicrob Agents Chemother 50, 3457-3459.
Oliveira, D. C., Milheirico, C., Vinga, S. \& de Lencastre, H. (2006b). Assessment of allelic variation in the $c c r A B$ locus in methicillinresistant Staphylococcus aureus clones. J Antimicrob Chemother 58, 2330 .

Shore, A. C., Rossney, A. S., O'Connell, B., Herra, C. M., Sullivan, D. J., Humphreys, H. \& Coleman, D. C. (2008). Detection of staphylococcal cassette chromosome mec-associated DNA segments in multiresistant methicillin-susceptible Staphylococcus aureus (MSSA) and identification of Staphylococcus epidermidis ccrAB4 in both methicillin-resistant S. aureus and MSSA. Antimicrob Agents Chemother 52, 4407-4419.

Stephens, A. J., Huygens, F., Inman-Bamber, J., Price, E. P., Nimmo, G. R., Schooneveldt, J., Munckhof, W. \& Giffard, P. M. (2006). Methicillin-resistant Staphylococcus aureus genotyping using a small set of polymorphisms. J Med Microbiol 55, 43-51.

Stephens, A. J., Huygens, F. \& Giffard, P. M. (2007). Systematic derivation of marker sets for staphylococcal cassette chromosome mec typing. Antimicrob Agents Chemother 51, 2954-2964.

Takano, T., Higuchi, W., Otsuka, T., Baranovich, T., Enany, S., Saito, K., Isobe, H., Dohmae, S., Ozaki, K. \& other authors (2008). Novel characteristics of community-acquired methicillin-resistant Staphylococcus aureus strains belonging to multilocus sequence type 59 in Taiwan. Antimicrob Agents Chemother 52, 837-845.

Wang, Q., Kong, F., Jelfs, P. \& Gilbert, G. L. (2008). Extended phage locus typing of Salmonella enterica serovar Typhimurium, using multiplex PCR-based reverse line blot hybridization. J Med Microbiol 57, 827-838.

Zhang, K., McClure, J. A., Elsayed, S., Louie, T. \& Conly, J. M. (2005). Novel multiplex PCR assay for characterization and concomitant subtyping of staphylococcal cassette chromosome mec types I to $\mathrm{V}$ in methicillin-resistant Staphylococcus aureus. J Clin Microbiol 43, 50265033. 